J. Gynäkol. Endokrinol. CH 2021 · 24:1 https://doi.org/10.1007/s41975-021-00189-w Angenommen: 4. März 2021

Online publiziert: 23. März 2021

(c) Springer-Verlag GmbH Austria, ein Teil von Springer Nature 2021

\section{Liebe Kolleginnen und Kollegen,}

der Frühling naht, und seit vier Tagen haben die Geschäfte in der Schweiz wieder geöffnet. Das wird bei vielen Glücksgefühle auslösen, die nicht zuletzt mit einem Anstieg des Neurotransmitters Serotonin assoziiert sind. Aber woher kommt das Serotonin? Und welche Folgen hat eine Störung des Tryptophanhaushalts? Hierzu werden Sie viel Wissenswertes und praktisch Umsetzbares im Artikel von M. Passarge \& B. Schütz finden.

So hat Tryptophan u.a. einen Einfluss auf unser Immunsystem, und gerade Frauen sind häufiger als Männer von Autoimmunerkrankungen betroffen. $\mathrm{Zu}$ den häufigen Autoimmunerkrankungen bei Frauen zählen die Autoimmunthyreopathien, v. a. die Hashimoto-Thyreoiditis. Und hier stellt sich oft die Frage, welcher TSH-Serumspiegel anzustreben bzw. behandlungsbedürftig ist. Auf diese Frage geht der Artikel von A. Mondorf et al. ein, im Speziellen darauf, wie die Schilddrüsenfunktion in den Wechseljahren beurteilt und therapiert werden sollte.

Mit dem Älterwerden, v. a. mit dem Eintritt der Menopause, steigt das Osteoporoserisiko der Frau. Da die Osteoporose eine schleichende Erkrankung ist, gerät sie manchmal in Vergessenheit. Deswegen ist es so wichtig, dass wir sie als Gynäkologen/-innen auf dem Radar haben. Letztes Jahr hat die Fachgesellschaft SVGO ein Update ihrer Osteoporoseempfehlungen publiziert. Dies war Anlass, das Management der Knochengesundheit zu rekapitulieren (P. Stute \& C. Meier).

Das Thema Osteoporose ist aber nicht nur ein Thema der älteren Frau! Auch in der Adoleszenz oder im jungen Er-

\title{
Petra Stute
}

Abteilung für Gynäkologische Endokrinologie und Reproduktionsmedizin, Universitätsklinik für Frauenheilkunde, Inselspital Bern, Bern, Schweiz

\section{Editorial}

wachsenenalter können die Knochen leiden, z. B. wenn ein Östrogenmangel aufgrund einer Essstörung oder von Leistungssport auftritt. Bisher haben wir bei den Folgen des Leistungssports v.a. an die Mädchen/Frauen gedacht und die hormonelle Dysregulation „female athlete triad“ (FAT) genannt. FAT ist eine Kombination aus Amenorrhö sowie verminderter Knochendichte und Energieverfügbarkeit. Da aber selbstverständlich auch Athleten von einer reduzierten Energieverfügbarkeit betroffen sein können, wurde der Begriff nun - gendergerecht - in RED-S („relative energy deficiency in sports") umgewandelt. In ihrem Artikel geht S. Baumgartner auf das Management von RED-S bei Mädchen/Frauen ein.

Ich wünsche Ihnen beim Lesen viele neue interessante Erkenntnisse! Halten Sie Ihren Serotonin- und Vitamin-DSpiegel hoch genug, um den Lockdown und eine evtl. Coronainfektion möglichst gut zu überstehen!

Ihre Petra Stute

PS: Jetzt Mitglied im kostenlosen GEF Netzwerk werden und Sie erhalten regelmässig Neuigkeiten aus dem Gebiet der gynäkologischen Endokrinologie. Einfach anmelden unter: gynendofreunde@ gmail.com

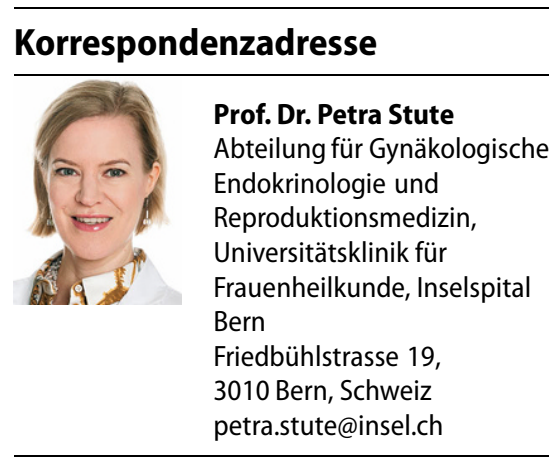

Interessenkonflikt. P. Stute gibt an, dass kein Interessenkonflikt besteht.

Hinweis des Verlags. Der Verlag bleibt in Hinblick auf geografische Zuordnungen und Gebietsbezeichnungen in veröffentlichten Karten und Institutsadressen neutral. 